\title{
Concomittant Coronary Revascularization and Resection of a Cardiac Tumor
}

\author{
Denyan Mansuroglu, Kenan Sever, Murat Ugurlucan ${ }^{*}$ and Yelda Saltan
}

Gaziosmanpasa Hospital, Cardiovascular Surgery Clinic, Istanbul, Turkey

\begin{abstract}
Ischemic cardiac diseases are the leading causes of mortality in the developed and developing regions of the world. Although vascular problems may accompany coronary artery disease, association of primary cardiac tumors with ischemic heart disease and the treatment of the combination are rarely reported in the literature. This report presents a male patient who successfully underwent concomitant coronary artery bypass grafting with resection of right atrial myxoma.

In this report, we present a case of ischemic heart disease and myxoma treated successfully in the same surgical session.
\end{abstract}

Keywords: Ischemic heart disease, coronary artery bypass grafting, cardiac myxoma.

\section{INTRODUCTION}

Coronary artery diseases, although in the past 10 years were proposed to account for the major aetiology of mortality in the developed countries, the pathology, today, are pandemic and are one of the leading causes of mortality, morbidity and high medical expenses. Since the beginning of cardiac surgery, despite advances in anesthesiology, surgery and medical management policies, coronary revascularization, i.e. coronary artery bypass grafting (CABG), still accounts for the most realistic solution in the treatment of the ischemic heart disease [1].

Myxomas are the most common tumors seen in the heart and mostly located in the left atrium originating from the atrial septum [2]. Cases are usually sporadic. Although there is not a proven association between ischemic heart disease and myxomas, the reported incidence of coronary artery disease in patients with cardiac myxomas range between 7 $12 \%[3]$.

In this report, we present a patient with right atrial myxoma and ischemic heart disease who underwent successful concomitant treatment of both pathologies.

\section{CASE REPORT}

The patient was a 49-year-old male presented to the clinic with chest pain on exertion, which progressed in the last three months. In his history, there were two packs of cigarette smoking/day for 25 years. Family history was not remarkable. Physical examination revealed grade 2-3/6 diastolic murmur otherwise completely normal. There were anemia (hematocrit level of 27.3\%), elevated erythrocyte sedimentation rate $(38 \mathrm{~mm} /$ hour $)$, C-reactive protein (44.09mg/L, Normal: $0-6 \mathrm{mg} / \mathrm{L})$ and fasting blood glucose $(132 \mathrm{mg} / \mathrm{dl}$, Normal: $70-100 \mathrm{mg} / \mathrm{dl})$ in routine laboratory work

*Address correspondence to this author at the Bozkurt Caddesi, No: 110112, Benli Apt., Daire:9, 80250 Kurtulus, Istanbul, Turkey; Tel: +90 535 43167 86; Fax: +90 21223525 68; E-mail: muratugurlucan@yahoo.com up. Chest X-ray indicated increased cardiothoracic index. Stress electrocardiography was positive and it was suggestive for myocardial ischemia at the left anterior descending coronary artery territory. Echocardiography showed a $6 \times 6.5 \mathrm{~cm}$ in diameter tumoral mass, most probably a myxoma, originating from the right atrial wall (Fig. 1), moving freely during systole and diastole and leading to grade 23 tricuspid regurgitation. There was patent foramen ovale. Right atrium and ventricle were enlarged and pulmonary artery pressure was increased to $45 \mathrm{mmHg}$. Ejection fraction was $50 \%$. The patient underwent coronary angiography, which revealed triple vessels coronary artery disease $(80 \%$ stenosis in the left anterior descending artery, $85 \%$ stenosis in the circumflex coronary artery and $70 \%$ stenosis in the right coronary artery).

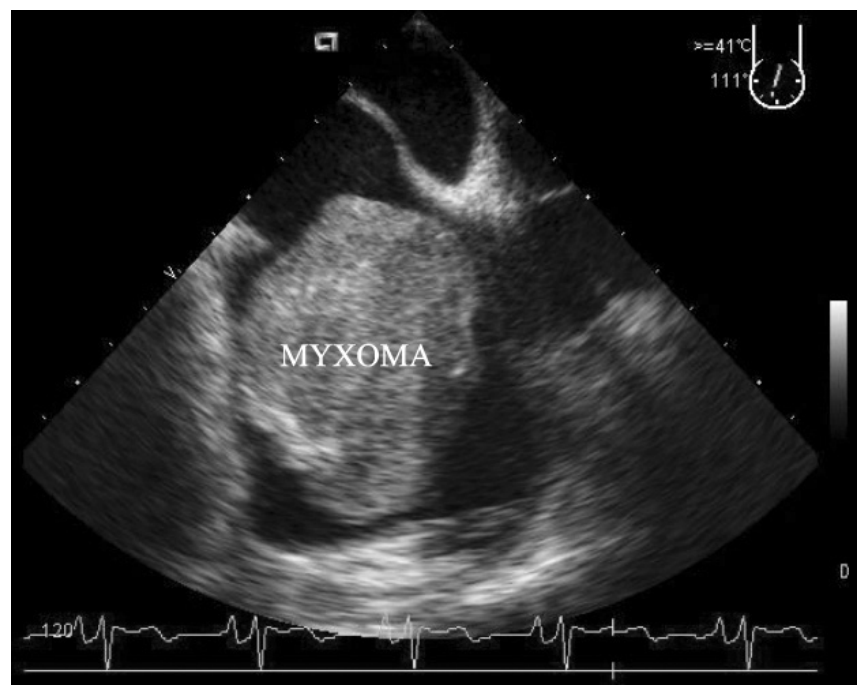

Fig. (1). Echocardiographic view of the myxoma measuring $6 \times 6.5 \mathrm{~cm}$ in diameter originating from the right atrial wall and filling the right atrial cavity.

Following detailed explanation of the pathology itself and risks and benefits of the concomitant and two stage 
surgeries to the patient, the informed consent was obtained and the patient underwent surgical treatment. Through median sternotomy, aortic and bicaval cannulations were performed. Cardiac arrest was fashioned with ante grade cold blood cardioplegia. Following snaring of both vena cavae, right atriotomy was performed through an incision parallel to the Waterston groove. Myxoma was inspected to originate from the wall of the appendix of the right atrium and filled the right atrial cavity extensively (Fig. 2). Tumor was resected together with the wall of the right atrial appendix. Patent foramen ovale was sutured primarily. Tricuspid valve coaptation was checked with saline flush and found to be mild. Right atriotomy was closed and CABG was performed with the anastomosis of left internal thoracic artery to left anterior descending artery, and with two separate saphenous vein grafts to first obtuse marginal artery and posterior descending artery. Postoperative course was uneventful and the patient was discharged from the hospital on the sixth postoperative day. Histopathologic examination of the resected mass revealed tumor rich in glycosaminoglycan and composed of polygonal myxoma cells confirming the pre- and perioperative diagnoses. The patient was followed up free of symptoms for more than nine months.

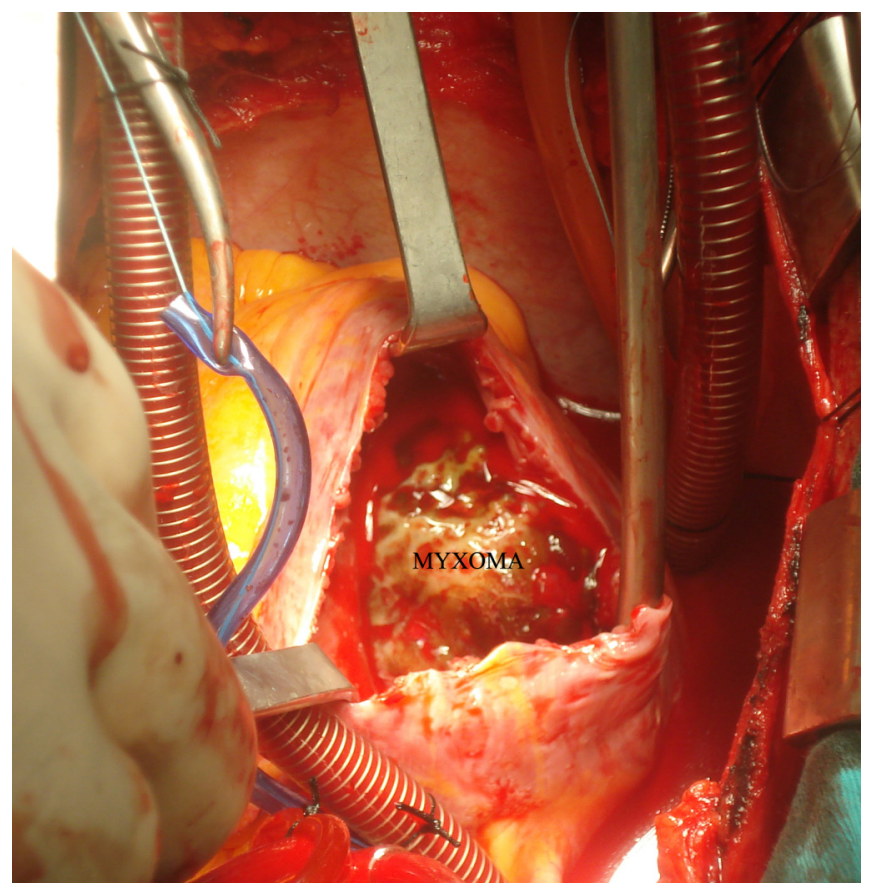

Fig. (2). Perioperative view of the right atrial myxoma.

\section{DISCUSSION}

Tumors originating from the myocardium are very rare with a reported incidence of 1,7-10/100,000. They may occur at all age groups. They have a benign course in $65-80 \%$ of the cases [4]. Since the first successful resection of a left artrial myxoma by Crafoord in 1954, myxomas are successfully diagnosed and treated with the advances in diagnostic tools, surgical and anesthesiologic measures in the current era [5].

Myxomas are usually seen in the 3rd-6th decades of life and more frequent among women [4]. Early diagnosis and treatment are vital to prevent valvular pathologies in the heart, as well as thromboembolic complications secondary to the tumor [4-6].

The most frequently encountered location for cardiac myxomas is the left atrium (75\%) followed by right atrium $(15-20 \%)$ and both ventricles (6-8\%). Symptoms secondary to the tumor are generally non-specific and short lasting such as fever, weight loss, fatigue, palpitation, dyspnoea ... etc. More specifically, they may obstruct the blood flow inside the heart, which in turn may lead to hemodynamic instability. Syncope and rarely lethal events secondary to flow stuck inside the heart have also been reported in the literature. They may lead to valvular pathologies including stenosis or regurgitation as well as permanent valve deformities. Overall, signs of the pathology itself are related to the association of the tumor and its intracardiac location [2-5]. More specifically, auscultation findings such as tumor "plop" or heart murmur altering with the position of the patient are suggestive for myxomas [6]. Other than intracardiac consequences, myxomas may lead to systemic or pulmonary embolism according to the location of the tumor. Laboratory findings in the presence of myxomas are again nonspecific including leucocytosis, elevated erythrocyte sedimentation rate and cyaloreactive protein, anemia, thrombocytopenia, ...etc [4].

Cardiac myxomas can easily be diagnosed by echocardiography or advanced cardiac imaging models such as cardiac magnetic resonance imaging or computerized tomography [7]. Detailed information about the tumor, such as the location, size, mobility, attachment features can easily be predicted by cheaper conventional transesophageal or transthoracic echocardiography measures [8]. Cardiac catheterization is reserved for patients with suspected ischemic heart diseases or for patients above certain ages. Moreover, intracardiac flow or pressure measurement studies should be avoided to prevent iatrogenic embolism during angiography [6].

Treatment of myxomas is performed by surgical resection of the mass as soon as the diagnosis is made to prevent occurrence of intracardiac deformities or complications [28]. Delay in treatment is highly associated with embolic events. Surgical treatment should reveal complete resection of the tumor with tumor free margins otherwise, recurrence may be seen in the postoperative follow up period. Associated cardiac pathologies such as valvular or ischemic heart diseases are also treated ideally and accordingly in the same surgical session.

On the other hand, a definitive relationship between atrial myxomas and coronary artery disease has not been identified. Sugimoto et al. [9] detected ischemic heart disease in $9.5 \%$ of the patients in their 21 patient series with atrial myxomas. In another study by Cleemput et al. [10] the incidence of concomitant coronary artery disease and atrial myxoma was diagnosed as $10.5 \%$. Especially after the age of 35 , the co-incidence of atrial myxomas with ischemic heart disease without an evidenced etiopathology increases [11]. Very rarely, in patients with atrial myxoma presenting with myocardial infarction, it has been shown that the infarction resulted from the embolization particles from the pedicle of the myxoma [12,13]. Nevertheless, when both disorders account concomitantly, ideal treatment should include same session surgery. 
In conclusion, although cardiac myxomas and ischemic heart diseases are well-known cardiosurgical problems, combination of both pathologies and concomitant treatment are rarely presented in the literature. Additionally, cardiac myxomas commonly originate from interatrial septum and place in the left atrium; however, in our male patient the lesion took place in the right atrium originating from the right atrial free wall.

\section{ACKNOWLEDGEMENT}

Authors would like to thank Ms. Toni Spring for the linguistic revision of the manuscript.

\section{REFERENCES}

[1] Furlani D, Klopsch C, Gäbel R, et al. Intracardiac erythropoietin injection reveals antiinflammatory potential and improved cardiac functions detected by Forced Swim Test. Transplant Proc 2008; 40(4): 962-6.

[2] Kejriwal NK, Tan J, Ullal RR, Alvarez JM. Atrial myxoma with coexistent coronary artery disease: a report of two cases. Heart Lung Circ 2003; 12(2): 108-11.

[3] Burke A, Li L, Kling E, Kutys R, Virmani R, Miettinen M. Cardiac inflammatory myofibroblastic tumor: a "benign" neoplasm that may result in syncope, myocardial infarction, and sudden death. Am J Surg Pathol 2007; 31(7): 1115-22.

[4] Tansel T, Harmandar B, Ugurlucan M, et al. Over 14 years of experience with cardiac myxomas. Acta Cardiol 2006; 61(3): 285-8.

[5] D'Alfonso A, Catania S, Pierri MD, et al. Atrial myxoma: a 25-year single-institutional follow-up study. J Cardiovasc Med (Hagerstown) 2008; 9(2): 178-81.

[6] Ekmektzoglou KA, Samelis GF, Xanthos T. Heart and tumors: location, metastasis, clinical manifestations, diagnostic approaches and therapeutic considerations. J Cardiovasc Med (Hagerstown) 2008; 9(8): $769-77$.

[7] Gavrielatos G, Letsas KP, Pappas LK, et al. Large left atrial myxoma presented as fever of unknown origin: a challenging diagnosis and a review of the literature. Cardiovasc Pathol 2007; 16(6): 365-7.

[8] Kuon E, Kreplin M, Weiss W, Dahm JB. The challenge presented by right atrial myxoma. Herz 2004; 29(7): 702-9.

[9] Sugimoto T, Ogawa K, Asada T, et al. Surgical treatment of left atrial myxoma with coronary arterial lesion. Kyobu Geka 1993; 46(2): 155-9.

[10] Van Cleemput J, Daenen W, De Geest H. Coronary angiography in cardiac myxomas: findings in 19 consecutive cases and review of the literature. Cathet Cardiovasc Diagn 1993; 29(3): 217-20.

[11] Rice PL, Pifarré R. Left atrial myxoma and coronary artery disease: combined surgical treatment. Arch Surg 1981;116(3): 353-5.

[12] Soejima Y, Niwa A, Tanaka M, et al. A left atrial myxoma complicated with acute myocardial infarction. Intern Med 1997; 36(1): 31-4.

[13] Panos A, Kalangos A, Sztajzel J. Left atrial myxoma presenting with myocardial infarction. Case report and review of the literature. Int $\mathrm{J}$ Cardiol 1997; 62(1): 73-5. 